increasing high-density lipoprotein. Am J Cardiol. 2006;98(11):1542-1549.

4. Williams KJ, Feig JE, Fisher EA. Rapid regression of atherosclerosis: insights from the clinical and experimental literature. Nat Clin Pract Cardiovasc Med. 2008;5(2):91-102.

5. Mendez AJ. The promise of apolipoprotein A-I mimetics. Curr Opin Endocrinol Diabetes Obes. 2010;17(2):171-176.

6. Khera AV, Rader DJ. Discovery and validation of new molecular targets in treating dyslipidemia: the role of human genetics. Trends Cardiovasc Med. 2009;19(6):195-201.

7. Briel M, et al. Association between change in high density lipoprotein cholesterol and cardiovascular disease morbidity and mortality: systematic review and meta-regression analysis. BMJ. 2009;338:b92.

8. Singh IM, Shishehbor MH, Ansell BJ. High-density lipoprotein as a therapeutic target: a systematic review. JAMA. 2007;298(7):786-798.

9. Barter PJ, et al. Effects of torcetrapib in patients at high risk for coronary events. $N$ Engl J Med. 2007;357(21):2109-2122.

10. Besler C, et al. Mechanisms underlying adverse effects of HDL on eNOS-activating pathways in patients with coronary artery disease. J Clin Invest. 2011;121(7):2693-2708.

11. Duffy D, Rader DJ. Update on strategies to increase HDL quantity and function. Nat Rev Cardiol. 2009;6(7):455-463.

12. Khera AV, et al. Cholesterol efflux capacity, highdensity lipoprotein function, and atherosclerosis. N Engl J Med. 2011;364(2):127-135

13. Mineo C, Deguchi H, Griffin JH, Shaul PW. Endothelial and antithrombotic actions of HDL. Circ Res. 2006;98(11):1352-1364.

14. Yuhanna IS, et al. High-density lipoprotein binding to scavenger receptor-BI activates endothelial nitric oxide synthase. Nat Med. 2001;7(7):853-857.

15 . Seetharam D, et al. High-density lipoprotein promotes endothelial cell migration and reendothelialization via scavenger receptor-B type I. Circ Res.
2006;98(1):63-72

16. Saddar S, Mineo C, Shaul PW. Signaling by the highaffinity HDL receptor scavenger receptor B type I. Arterioscler Thromb Vasc Biol. 2010;30(2):144-150.

17. Assanasen C, et al. Cholesterol binding, efflux, and a PDZ-interacting domain of scavenger receptorBI mediate HDL-initiated signaling. J Clin Invest. 2005;115(4):969-977.

18. Yancey PG, et al. High density lipoprotein phospholipid composition is a major determinant of the bidirectional flux and net movement of cellular free cholesterol mediated by scavenger receptor BI. J Biol Chem. 2000;275(47):36596-36604

19. Shao B, et al. Modifying apolipoprotein A-I by malondialdehyde, but not by an array of other reactive carbonyls, blocks cholesterol efflux by the ABCA1 pathway. J Biol Chem. 2010;285(24):18473-18484.

20. Regieli JJ, et al. Paraoxonase variants relate to 10 -year risk in coronary artery disease: impact of a high-density lipoprotein-bound antioxidant in secondary prevention. J Am Coll Cardiol. 2009;54(14):1238-1245.

\title{
Adenosine: front and center in linking nutrition and metabolism to neuronal activity
}

\author{
Robert W. Greene
}

Department of Psychiatry, University of Texas Southwestern Medical Center and Dallas VA Medical Center, Dallas, Texas, USA.

\begin{abstract}
Many individuals with epilepsy benefit from consuming a ketogenic diet, which is similar to the more commonly known Atkins diet. The underlying molecular reason for this has not been determined. However, in this issue of the JCI, Masino et al. have elucidated the mechanism responsible for the antiepileptic effects of the ketogenic diet in mice. The diet is shown to decrease expression of the enzyme adenosine kinase (Adk), which is responsible for clearing the endogenous antiepileptic agent adenosine (Ado) from the extracellular CNS space. Decreased expression of Adk results in increased extracellular Ado, activation of inhibitory Ado $A_{1}$ receptors, and decreased seizure generation, the desired therapeutic effect. The authors' work serves to emphasize the importance of controlling Adk expression, not only as the mechanism of action of the ketogenic diet, but also as a potential target of future therapies.
\end{abstract}

Epilepsy is a brain disorder characterized by recurrent seizures that last a few seconds to up to 15 minutes, but seldom longer. The seizures are a result of synchronized electrical discharge by a large proportion of CNS neurons. They usually originate from one or more hyperexcitable neuronal foci and propagate to both surrounding neuronal tissue and downstream targets of the excited neurons. Most therapeutic approaches involve pharmacological agents targeted toward reducing the excitability of the irritable seizure focus and/or the propagation of the synchronized discharges. However,

Conflict of interest: The author has declared that no conflict of interest exists.

Citation for this article: J Clin Invest. doi:10.1172/ JCI58391. these agents often cause undesirable side effects, and in some individuals with epilepsy, the seizures are refractory to pharmacological agents. An alternative therapy that has shown some success in this regard is the ketogenic diet.

The ketogenic diet, similar to the more commonly known Atkins diet, provides a metabolic treatment for epilepsy. The highfat, low-carbohydrate diet forces ketonebased rather than glucose-based metabolism and has well-demonstrated antiepileptic efficacy (1). However, the mechanism responsible for the anticonvulsant effect of a ketogenic diet remains to be characterized. In this issue of the JCI, Masino et al. convincingly demonstrate that a ketogenic diet works by modifying the availability of one of the best-characterized and effica- cious endogenous antiepileptic compounds in mammals, adenosine (Ado) (2). The authors show that in mice, the ketogenic diet is associated with decreased expression of the enzyme responsible for removal of Ado from the CSF, adenosine kinase (Adk). As a result, there is an increase in Ado in the CSF that in turn activates Ado $A_{1}$ receptors $\left(\right.$ Ado $\left._{1} R s\right)$. This activation was found to be both necessary and sufficient for the antiepileptic effect of the ketogenic diet. The work of Masino et al. (2) serves to emphasize the therapeutic potential of controlling Adk expression in epilepsy and the need for better understanding of this control.

\section{Ado: the endogenous antiepileptic agent}

An endogenous antiepileptic agent should have, at the least, the following two properties. First, its levels should be increased in brain tissue by conditions that predispose to seizure generation and/or by seizure activity itself. Second, when released, it should act to reduce the likelihood of the seizure activity.

Conditions that promote seizures include hypoxia of nervous tissue; hypoglycemia; increased levels of potassium or other excitogenic substances, such as glutamate (for example, as might be released as a result of acute brain trauma), in the brain extracellular medium; or simply an 


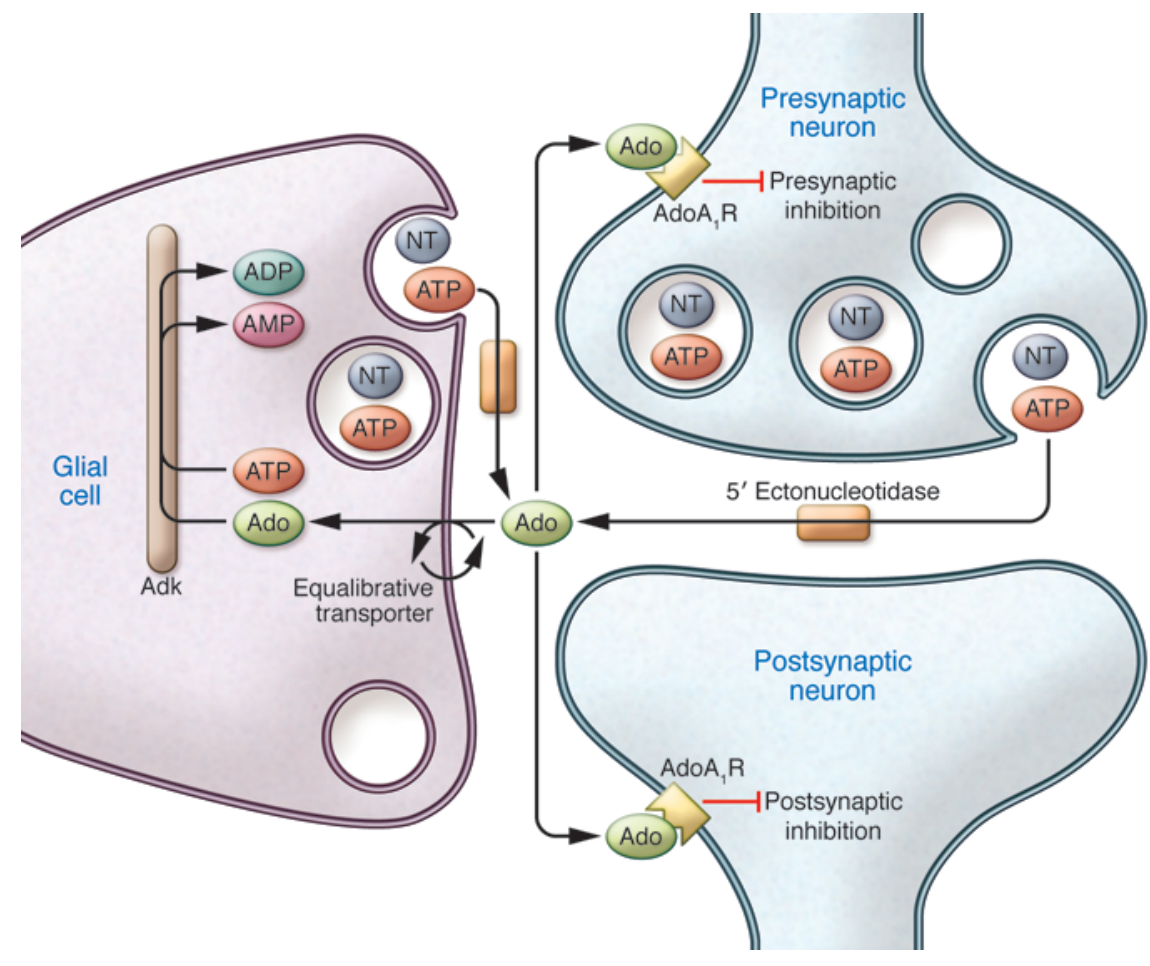

Figure 1

CNS pathways for Ado metabolism. Under physiological conditions, Ado is coreleased from neuronal and glial neurotransmitter (NT) vesicles as ATP. The ATP is broken down to Ado by $5^{\prime}$ ectonucleotidases. Ado flows down its concentration gradient into glia, facilitated by equilibrative transporters (primarily nitrobenzylthiolionosine-sensitive transporter), where it is metabolized together with ATP to AMP and ADP by high-affinity, low-capacity Adk. A change in glial intracellular Ado may occur with a reduction in levels of glial Adk, as a result of maintaining a ketogenic diet. The glial intracellular increase in Ado is reflected extracellularly, where Ado can activate pre- and postsynaptic $\operatorname{Ado}_{1} R$ to increase Ado-mediated inhibitory tone and exert an antiepileptic effect.

abnormally high level of neural activity. Each of these epileptogenic conditions increases the excitability of the neural tissue and is known to trigger a large release of Ado into the extracellular medium of brain tissue, as does the increased neural activity of the seizure itself (3).

Extracellular Ado may activate $\mathrm{AdoA}_{1} \mathrm{Rs}$ to cause (a) presynaptic inhibition; (b) increased postsynaptic $G$ protein inwardly rectifying potassium (GIRK) conductance; and (c) decreased hyperpolarization-activated current. All three effects decrease neuronal excitability (reviewed in refs. 4, 5), which decreases the likelihood of seizure generation or propagation.

\section{Ado-mediated homeostasis between CNS metabolic state and CNS neural excitability}

The conditions resulting in increased CNS extracellular Ado and the inhibitory tone resulting from Ado activation of $\mathrm{Ado}_{1}$ Rs have led to the suggestion that Ado mediates negative feedback to maintain a healthy homeostasis between the metabolic state of brain tissue and the electrophysiological excitability of nervous tissue $(4,6-8)$. As metabolite availability decreases in the CNS (for example, as a result of brain hypoxia or hypoglycemia), or as neural energy demand increases (for example, because of increased levels of potassium or glutamate in the brain extracellular medium or increased neural activity, including seizure activity), Ado levels increase extracellularly. In turn, Ado mediates homeoexcitability via activation of the inhibitory Ado $_{1}$ Rs. This homeostatic control may be extended to physiological conditions at glutamate synapses. It has been shown that synaptically released glutamate, through activation of NMDA receptors, causes Ado release that feeds back onto glutamate presynaptic $\mathrm{Ado}_{1} \mathrm{Rs}$, inhibiting presynaptic static negative feedback to reduce neural release of glutamate and closing the synaptic glutamate/Ado homeostatic loop (9). Ado may thus mediate a tonic inhibitory control under both physiologic and pathologic conditions (10-12).

\section{Metabolism of Ado in the CNS}

Under baseline conditions, the majority of extracellular Ado originates from the breakdown of synaptosomally released ATP by $5^{\prime}$ ectonucleotidases (13). Presumably, the vast majority of ATP derives from neuronal release, but glia may provide a significant source of extracellular ATP though vesicular release of the nucleotide (Figure 1 and ref. 14). Extracellular Ado fluxes down its concentration gradient into glia and neurons, facilitated by glial and neuronal nitrobenzylthiolionosinesensitive equilibrative transporters (15). Because the equilibrative transporters depend on intracellular metabolism of Ado to maintain inward flux of Ado, the velocity of the intracellular Ado metabolizing enzymes ultimately determines the rate of removal of Ado from the extracellular medium. Hence, given a steady-state rate of production, the extracellular Ado concentration is determined by the rate of intracellular Ado metabolism.

The metabolic enzyme with the highest affinity for Ado is Adk, but it has a low capacity in the brain. Under most physiologic conditions, the $V_{\max }$ of Adk appears to determine the Ado rate of metabolism (16) and, accordingly, the extracellular concentration of Ado in nervous tissue. Nonetheless, because the mechanism of action of Adk involves binding to ATP and Ado, transfer of a phosphate from ATP to Ado, and subsequent production of AMP and ADP, the ATP/ADP ratio affects its velocity (17). This becomes particularly relevant in light of the fact that Adk is localized in the adult mammalian CNS to glia (18). Ado control of CNS neuronal excitability is therefore modulated by the ATP/ADP ratio of glia and their metabolic state. Ado-mediated inhibitory tone can also be controlled by the level of Adk expression because under most conditions, the $V_{\max }$ of Adk appears to be the rate-limiting factor for Ado metabolism $(15,19,20)$. This is borne out by pharmacological blockade of Adk, which potently increases Ado efflux in association with increased Ado $_{1}$ R-mediated inhibitory tone in the hippocampus (21) and in cholinergic arousal centers (22). Of note, little is known about how Adk expression is controlled other than 
as a consequence of increased numbers of Adk-expressing cells, as happens with astrogliosis (23).

\section{Mechanism for the antiepileptic effects of ketogenic diet}

Masino et al. provide evidence that the ketogenic diet decreases Adk expression and that its antiepileptic effect is dependent on the resulting increased Ado activation of $A_{d o} A_{1} R$ (2). The study is one of the first to show that environmental influences - in this case, ketogenic diet - can decrease Adk expression. The authors showed that the antiepileptic effect of this Adk decrease is dependent on resulting Ado activation of $A_{d o} A_{1} R$ : first, by reducing seizures in epileptic mice fed a ketogenic diet; and second, by restoring the seizures through pharmacological blockade of $\mathrm{Ado}_{1} \mathrm{R}$. Finally, in Ado $A_{1}$ R-knockout mice, genetically engineered so that either levels of Adk cannot be reduced or there are no $\mathrm{Ado}_{1} \mathrm{Rs}$, the ketogenic diet was shown to have no antiseizure effect. This provides a mechanistic rationale for the clinical practice of counseling patients with epilepsy to avoid caffeine. This widely used stimulant is an antagonist of $\mathrm{AdoA}_{1} \mathrm{R}$ (and also of $\mathrm{AdoA}_{2} \mathrm{R}$ ) and is usually avoided by patients with epilepsy, especially by those treated with ketogenic diet. These findings are in keeping with earlier studies using pharmacological blockade of Adk to potently prevent seizure activity in vivo in rats (20). They are also consistent with the seizure-promoting effects in mice of brain overexpression of Adk (resulting from either transgenic expression or increased Adk expression secondary to gliosis), as demonstrated by Boison and colleagues $(23,24)$.

An interesting observation from the Masino et al. study that is relevant to acute Adk activity (i.e., the velocity of Adk given its acute level of expression) is that acute administration of glucose increases seizure activity almost to pre-ketogenic diet levels (2). This can be understood as an acute glucose-stimulated increase in ATP that drives the $\mathrm{ATP}+\mathrm{Ado} \rightarrow \mathrm{ADP}+\mathrm{AMP}$ Adk-catalyzed reaction to decrease Ado. The result is that the change in glial metabolic state can rapidly decrease intracellular Ado (and hence extracellular Ado, by the equilibrative transport system) to increase seizure activity.

\section{Translation of the mouse models to the clinic}

Masino et al. use seizure models that involve only Adk overexpression or only loss of $\mathrm{Ado}_{1} \mathrm{R}$ expression (2). As mentioned above, pharmacologic blockade of Adk exerts a powerful antiepileptic effect in other models of epilepsy, and it will be important to show the effectiveness of the ketogenic diet in these other models. In fact, even in the present study, the seizure activity shown with Adk and $\operatorname{Ado}_{1}$ R mutants is likely to result from a combination of the Ado-related seizure-promoting mutations and the injury secondary to the placement of depth electrodes in the hippocampus. We have recorded many hundreds of hours of temporal lobe EEG activity in mice lacking $\mathrm{Ado}_{1} \mathrm{R}$ only in excitatory neurons and have never observed any seizure-like activity (25). It seems likely that the seizure activity reported by Masino et al. (2) is caused, at least in part, by the depth electrodes.

The authors' conclusion provides not only important insight into the mechanism of the antiepileptic action of the ketogenic diet, but also important understanding of the relationship between the metabolic state of glia and the Ado-mediated inhibitory tone exerted on most of the forebrain nervous system. The effects of increased inhibitory tone and inhibited seizures were shown to be strikingly sensitive to Adk expression level, which was shown to be downregulated by the ketogenic diet. Future studies will undoubtedly seek to uncover the mechanism responsible for this important downregulation. A better understanding of these pathways may lead to more effective diets and/or pharmacological targets for future epilepsy therapies.

\section{Acknowledgments}

The work for this commentary was supported by the Department of Veterans Affairs.

Address correspondence to: Robert W. Greene, Department of Psychiatry, University of Texas Southwestern Medical Center and Dallas VA Medical Center, Dallas, Texas 75390, USA. Phone: 214.648.5108; Fax:214.648.7037; E-mail: robertw.greene@ utsouthwestern.edu.

1. Freeman JM, Kossoff EH, Hartman AL. The ketogenic diet: one decade later. Pediatrics. 2007; 119(3):535-543.

2. Masino SA, et al. A ketogenic diet suppresses seizures in mice through adenosine $\mathrm{A}_{1}$ receptors. JClin Invest. 2011;121(7):2679-2683.

3. McIlwain H, Pull I. Release of adenine derivatives on electrical stimulation of superfused tissues from the brain. J Physiol. 1972;221(1):9P-10P.

4. Greene RW, Haas HL. The electrophysiology of adenosine in the mammalian central nervous system. Prog Neurobiol. 1991;36(4):329-341.
5. Dunwiddie TV, Masino SA. The role and regulation of adenosine in the central nervous system. Annu Rev Neurosci. 2001;24:31-55.

6. Greene RW, Haas HL. Adenosine actions on CA1 pyramidal neurones in rat hippocampal slices. J Physiol. 1985;366:119-127.

7. Magistretti PJ, Hof PR, Martin JL. Adenosine stimulates glycogenolysis in mouse cerebral cortex: a possible coupling mechanism between neuronal activity and energy metabolism. J Neurosci. 1986;6(9):2558-2562.

8. McIlwain H, Poll JD. Adenosine in cerebral homeostatic role: appraisal through actions of homocysteine, colchicine, and dipyridamole. J Neurobiol. 1986;17(1):39-49.

9. Brambilla D, Chapman D, Greene R. Adenosine mediation of presynaptic feedback inhibition of glutamate release. Neuron. 2005;46(2):275-283.

10. Dunwiddie TV. Endogenously released adenosine regulates excitability in the in vitro hippocampus. Epilepsia. 1980;21(5):541-548.

11. Dunwiddie TV, Hoffer BJ. Adenine nucleotides and synaptic transmission in the in vitro rat hippocampus. BrJ Pharmacol. 1980;69(1):59-68.

12. Greene RW, Haas HL, Hermann A. Effects of caffeine on hippocampal pyramidal cells in vitro. $\mathrm{BrJ}$ Pharmacol. 1985;85(1):163-169.

13. MacDonald WF, White TD. Nature of extrasynaptosomal accumulation of endogenous adenosine evoked by $\mathrm{K}+$ and veratridine. J Neurochem. 1985;45(3):791-797.

14. Pascual $\mathrm{O}$, et al. Astrocytic purinergic signaling coordinates synaptic networks. Science. 2005; 310(5745):113-116

15. Sinclair CJ, Shepel PN, Geiger JD, Parkinson FE. Stimulation of nucleoside efflux and inhibition of adenosine kinase by $\mathrm{A} 1$ adenosine receptor activation. Biochem Pharmacol. 2000;59(5):477-483.

16. Arch JR, Newsholme EA. Activities and some properties of 5'-nucleotidase, adenosine kinase and adenosine deaminase in tissues from vertebrates and invertebrates in relation to the control of the concentration and the physiological role of adenosine. Biochem J. 1978;174(3):965-977.

17. Mimouni M, Bontemps F, Van den Berghe G. Kinetic studies of rat liver adenosine kinase. Explanation of exchange reaction between adenosine and AMP. J Biol Chem. 1994;269(27):17820-17825.

18. Studer FE, et al. Shift of adenosine kinase expression from neurons to astrocytes during postnatal development suggests dual functionality of the enzyme. Neuroscience. 2006;142(1):125-137.

19. Arch JR, Newsholme EA. The control of the metabolism and the hormonal role of adenosine. Essays Biochem. 1978;14:82-123.

20. Zhang G, Franklin PH, Murray TF. Manipulation of endogenous adenosine in the rat prepiriform cortex modulates seizure susceptibility.J Pharmacol Exp Ther. 1993;264(3):1415-1424.

21. Pak MA, Haas HL, Decking UK, Schrader J. Inhibition of adenosine kinase increases endogenous adenosine and depresses neuronal activity in hippocampal slices. Neuropharmacology. 1994;33(9):1049-1053.

22. Arrigoni E, Rainnie DG, McCarley RW, Greene RW. Adenosine-mediated presynaptic modulation of glutamatergic transmission in the laterodorsal tegmentum. J Neurosci. 2001;21(3):1076-1085.

23. Fedele DE, et al. Astrogliosis in epilepsy leads to overexpression of adenosine kinase, resulting in seizure aggravation. Brain. 2005;128(pt 10):2383-2395.

24. Pignataro G, Simon RP, Boison D. Transgenic overexpression of adenosine kinase aggravates cell death in ischemia. J Cereb Blood Flow Metab. 2007; 27(1):1-5.

25. Bjorness TE, Kelly CL, Gao T, Poffenberger V, Greene RW. Control and function of the homeostatic sleep response by adenosine A1 receptors. J Neurosci. 2009;29(5):1267-1276 